\title{
Path-integral formulation of pseudo-Hermitian quantum mechanics and the role of the metric operator
}

\author{
A. Mostafazadeh \\ Department of Mathematics, Koç University, Sariyer 34450, Istanbul, Turkey
}

(Received 7 June 2007; published 13 September 2007)

\begin{abstract}
We provide a careful analysis of the generating functional in the path-integral formulation of pseudoHermitian and in particular $\mathcal{P} \mathcal{T}$-symmetric quantum mechanics and show how the metric operator enters the expression for the generating functional.
\end{abstract}

DOI: 10.1103/PhysRevD.76.067701

PACS numbers: 03.65.Ca, 03.65.Db, 11.10.-z, 11.30.Er

In the pseudo-Hermitian representation of quantum mechanics [1], a quantum system is determined by a triplet $\left(\mathcal{H}, H, \eta_{+}\right)$where $\mathcal{H}$ is an auxiliary Hilbert space, $H: \mathcal{H} \rightarrow \mathcal{H}$ is a linear (Hamiltonian) operator with a real spectrum and a complete set of eigenvectors, and $\eta_{+}: \mathcal{H} \rightarrow \mathcal{H}$ is a linear, positive-definite, invertible (metric) operator fulfilling the pseudo-Hermiticity condition [2]

$$
H^{\dagger}=\eta_{+} H \eta_{+}^{-1} .
$$

The physical Hilbert space $\mathcal{H}_{\text {phys }}$ of the system is defined as the complete extension of the span of the eigenvectors of $\mathcal{H}$ endowed with the inner product

$$
\langle\cdot, \cdot\rangle_{+}:=\left\langle\cdot \mid \eta_{+} \cdot\right\rangle,
$$

where $\langle\cdot \mid \cdot\rangle$ is the defining inner product of $\mathcal{H}$, and the observables are identified with the self-adjoint operators acting in $\mathcal{H}_{\text {phys }}$, alternatively $\eta_{+}$-pseudo-Hermitian operators acting in $\mathcal{H}[1]$.

Pseudo-Hermitian quantum mechanics [1] has primarily developed in an attempt to unravel the mathematical structures responsible for the intriguing spectral properties of $\mathcal{P} \mathcal{T}$-symmetric Hamiltonians [3] such as

$$
H=\frac{p^{2}}{2 m}+\frac{\mu^{2} x^{2}}{2}+i \epsilon x^{3}, \quad m \in \mathbb{R}^{+}, \quad \mu, \epsilon \in \mathbb{R} .
$$

These properties were originally noticed in dealing with the field theoretical analogues of (3). It has also been conjectured that such models may have important applications in particle physics [4]. This has led a number of researchers to investigate various $\mathcal{P} \mathcal{T}$-symmetric field theories [5]. Similarly to standard quantum field theory, the path-integral techniques are indispensable in dealing with $\mathcal{P} \mathcal{T}$-symmetric field theories. Yet a careful analysis of the path-integral formulation of pseudo-Hermitian quantum mechanics has been lacking until recently. The first careful analysis of this problem is given in Ref. [6] where the authors use the Hermitian representation of the corresponding pseudo-Hermitian models to determine the appropriate source term in the generating functional $Z[J]$. The results of [6] give the impression that the metric operator, which is the central ingredient in the operator formulation of pseudo-Hermitian quantum mechanics, does not play an important role in the path-integral formulation of the theory. The purpose of the present paper is to elucidate the role of the metric operator in the latter formulation. This is indeed necessary for a correct formulation of $\mathcal{P} \mathcal{T}$-symmetric field theories. It also provides a straightforward interpretation of the results pertaining to the metric independence of thermodynamical quantities associated with noninteracting pseudo-Hermitian statistical mechanical systems that is discussed in [7].

Consider the definition of the generating functional (partition function) that is the starting point of path-integral formulation of quantum mechanics and quantum field theory:

$$
Z[J]:=\operatorname{tr}\left(\mathcal{T} \exp \left\{-\frac{i}{\hbar} \int_{t_{1}}^{t_{2}}(H-\vec{J} \cdot \vec{x}) d t\right\}\right),
$$

where "tr" stands for the trace, $\mathcal{T}$ exp is the time-ordered (chronological-ordered) exponential [8,9], $t_{1}$ and $t_{2}$ are initial and final time labels that in field theory are taken to be $-\infty$ and $\infty$, respectively, $\vec{J} \cdot \vec{x}=\sum_{i=1}^{m} J_{i} x_{i}$ is the source term, and $\vec{x}=\left(x_{1}, x_{2}, \cdots, x_{m}\right)$ is the dynamical configuration variable (field).

In standard quantum mechanics the source term $\vec{J} \cdot \vec{x}$ has a specific physical meaning (most conveniently explained within the context of Schwinger's variational principle [9]) and must be a physical observable. For the case that $\vec{J}$ is a real vector, which we consider here, this entails the dynamical variables $x_{i}$ to be physical observables ([9] pp. 166-167, DeWitt). In ordinary quantum mechanics this condition is satisfied because $x_{i}$ are Hermitian operators. But in pseudo-Hermitian quantum mechanics, one must use a specific metric operator to determine whether $x_{i}$ are physical observables. In the generic case, e.g., for the cubic oscillator (3), this is not the case. Therefore, the very definition of the generating functional needs to be modified as

$$
Z[J]:=\operatorname{tr}\left(\mathcal{T} \exp \left\{-\frac{i}{\hbar} \int_{t_{1}}^{t_{2}}(H-\vec{J} \cdot \vec{X}) d t\right\}\right),
$$

where $\vec{X}=\left(X_{1}, X_{2}, \cdots, X_{m}\right)$ are the pseudo-Hermitian dy- 
namical variables (position operators) [1]:

$$
X_{i}:=\eta_{+}^{-(1 / 2)} x_{i} \eta_{+}^{1 / 2} .
$$

Without the knowledge of $\eta_{+}$or the operator $Q:=$ $-\ln \eta_{+}$, one cannot construct $X_{i}$ and obtain the physically relevant generating functional for the theory. The generating functional (4) has no physical meaning unless $x_{i}$ commute with $\eta_{+}$(for all $i=1,2, \cdots, m$ ) or there are no external sources $(\vec{J}=\overrightarrow{0})$.

Consider the definition of tr that appears in (5). In the pseudo-Hermitian representation of the theory tr is defined by $[10]$

$$
\operatorname{tr}(A):=\sum_{n}\left\langle\psi_{n}, A \psi_{n}\right\rangle_{+}=\sum_{n}\left\langle\psi_{n} \mid \eta_{+} A \psi_{n}\right\rangle,
$$

where $\left\{\psi_{n}\right\}$ is an arbitrary orthonormal basis of the physical Hilbert space $\mathcal{H}_{\text {phys }}$ and $A$ is a trace-class operator acting in $\mathcal{H}_{\text {phys }}$. In view of the fact that $\eta_{+}^{1 / 2}: \mathcal{H}_{\text {phys }} \rightarrow \mathcal{H}$ is a unitary operator [11], we have

$$
\psi_{n}=\eta_{+}^{-(1 / 2)} \Psi_{n}
$$

for some orthonormal basis $\left\{\Psi_{n}\right\}$ of $\mathcal{H}$. Inserting (8) into (7) and making use of (2) and the Hermiticity of $\eta_{+}^{-(1 / 2)}$, we find

$$
\operatorname{tr}(A)=\sum_{n}\left\langle\Psi_{n} \mid \tilde{A} \Psi_{n}\right\rangle,
$$

where $\tilde{A}:=\eta_{+}^{1 / 2} A \eta_{+}^{-(1 / 2)}$. The right-hand side of (9) is precisely the trace of the operator $\tilde{A}$ that acts in $\mathcal{H}$. This calculation shows that the trace of a trace-class operator $A: \mathcal{H}_{\text {phys }} \rightarrow \mathcal{H}_{\text {phys }}$ is equal to the trace of its unitaryequivalent operator, namely $\tilde{A}: \mathcal{H} \rightarrow \mathcal{H}$;

$$
\operatorname{tr}(A)=\operatorname{tr}(\tilde{A}) .
$$

Next, we apply (10) for the case that $A=\mathcal{T} \exp \left\{-\frac{i}{\hbar} \times\right.$ $\left.\int_{t_{1}}^{t_{2}}(H-\vec{J} \cdot \vec{X}) d t\right\}$. Using (5) and (6), we have

$$
Z[J]=\operatorname{tr}\left(\mathcal{T} \exp \left\{-\frac{i}{\hbar} \int_{t_{1}}^{t_{2}}(h-\vec{J} \cdot \vec{x}) d t\right\}\right),
$$

where $h:=\eta_{+}^{1 / 2} H \eta_{+}^{-(1 / 2)}$ is the equivalent Hermitian Hamiltonian [11]. It is important to note that in the derivation of (11) we make an explicit use of the condition that $\eta_{+}$is time independent. This is a direct consequence of the projection axiom (measurement theory) and unitarity [12]. It allows us to establish the identity

$$
\begin{aligned}
\mathcal{T} & \exp \left\{-\frac{i}{\hbar} \int_{t_{1}}^{t_{2}}(h-\vec{J} \cdot \vec{x}) d t\right\} \\
& =\eta_{+}^{1 / 2} \mathcal{T} \exp \left\{-\frac{i}{\hbar} \int_{t_{1}}^{t_{2}}(H-\vec{J} \cdot \vec{X}) d t\right\} \eta_{+}^{-(1 / 2)}
\end{aligned}
$$

that in light of (10) leads to (11).

Equation (11) is an explicit manifestation of the physical equivalence of Hermitian and pseudo-Hermitian represen- tations of the system. It is this equivalence that is used in [6] to determine the form of the source term in the pathintegral expression for the generating functional.

Next, we consider the case that $H$ is time independent and that it has a real discrete spectrum and a complete set of eigenvectors. We can always choose a set of eigenvectors $\psi_{n}$ of $H$ and a set of eigenvectors $\phi_{n}$ of $H^{\dagger}$ such that $\psi_{n}$ and $\phi_{n}$ form a complete biorthonormal system, i.e.,

$$
\left\langle\psi_{n} \mid \phi_{m}\right\rangle=\delta_{m n}, \quad \sum_{n}\left|\phi_{n}\right\rangle\left\langle\psi_{n}\right|=1,
$$

and the metric operator $\eta_{+}$satisfies [13]

$$
\eta_{+}=\sum_{n}\left|\phi_{n}\right\rangle\left\langle\phi_{n}\right|
$$

In this case, $\left\{\psi_{n}\right\}$ form an orthonormal basis of $\mathcal{H}_{\text {phys }}, \eta_{+}$ maps $\psi_{n}$ to $\phi_{n}$, i.e.,

$$
\phi_{n}=\eta_{+} \psi_{n},
$$

and in view of (5), (7), and (14), and the identity $\int_{\mathbb{R}^{m}} d x_{1} d x_{2} \cdots d x_{m}|\vec{x}\rangle\langle\vec{x}|=1$, we have

$$
\begin{aligned}
Z[\overrightarrow{0}]= & \operatorname{tr}\left(e^{-\left(i\left(t_{2}-t_{1}\right) H / \hbar\right)}\right)=\sum_{n}\left\langle\psi_{n} \mid \eta_{+} e^{-\left(i\left(t_{2}-t_{1}\right) H / \hbar\right)} \psi_{n}\right\rangle \\
= & \sum_{n} \int_{\mathbb{R}^{m}} d x_{1} d x_{2} \cdots d x_{m}\left\langle\psi_{n}\left|\eta_{+}\right| \vec{x}\right\rangle \\
& \times\left\langle\vec{x} \mid e^{-\left(i\left(t_{2}-t_{1}\right) H / \hbar\right)} \psi_{n}\right\rangle \\
= & \int_{\mathbb{R}^{m}} d x_{1} d x_{2} \cdots d x_{m} \sum_{n}\left\langle\phi_{n} \mid \vec{x}\right\rangle\left\langle\vec{x} \mid e^{-\left(i\left(t_{2}-t_{1}\right) H / \hbar\right)} \psi_{n}\right\rangle \\
= & \int_{\mathbb{R}^{m}} d x_{1} d x_{2} \cdots d x_{m}\left\langle\vec{x}\left|e^{-\left(i\left(t_{2}-t_{1}\right) H / \hbar\right)} \sum_{n}\right| \psi_{n}\right\rangle\left\langle\phi_{n} \mid \vec{x}\right\rangle \\
= & \int_{\mathbb{R}^{m}} d x_{1} d x_{2} \cdots d x_{m}\left\langle\vec{x}\left|e^{-\left(i\left(t_{2}-t_{1}\right) H / \hbar\right)}\right| \vec{x}\right\rangle .
\end{aligned}
$$

This calculation shows that in the absence of an external source the generating functional does not depend on the choice of a metric operator. It is in this sense that the metric operator $\eta_{+}$or $Q=-\ln \eta_{+}$"disappears" from the calculations [6]. This phenomenon is the real reason for the metric independence of the thermodynamical quantities for the statistical systems considered in [7]. However, it does not extend to the case where one needs to couple the system to an external source.

As we explained above one cannot naively identify the source term with $\vec{J} \cdot \vec{x}$, generalize (15) to $Z[\vec{J}]=$ $\int_{\mathbb{R}^{m}} d x_{1} d x_{2} \cdots d x_{m}\left\langle\vec{x}\left|e^{-\left(i\left(t_{2}-t_{1}\right)(H-\vec{J} \cdot \vec{x}) / \hbar\right)}\right| \vec{x}\right\rangle$, and follow the usual root to a path-integral calculation of the generating functional and the corresponding $n$-point functions, as done, for example, in [14]. One must first make a choice for the metric operator $\eta_{+}$, determine the observable $\vec{X}$, define the generating functional according to (5), and repeat the derivation of (15) with the role of $H$ played by $H-\vec{J} \cdot \vec{X}$. This yields 


$$
Z[\vec{J}]=\int_{\mathbb{R}^{m}} d x_{1} d x_{2} \cdots d x_{m}\left\langle\vec{x}\left|e^{-i\left(\left(t_{2}-t_{1}\right) / \hbar\right)(H-\vec{J} \cdot \vec{X})}\right| \vec{x}\right\rangle .
$$

As seen from this expression the generating functional depends on the choice of the metric operator $\eta_{+}$, because $\vec{X}=\eta_{+}^{-(1 / 2)} \vec{x} \eta_{+}^{1 / 2}$. It is this particular metric dependence that makes the path-integral calculation of the generating functional a generally formidable task.

Unlike for the simple models considered in [6], in general the explicit form of an appropriate metric operator and the pseudo-Hermitian observables that couple to sources is not known. For example, a perturbative calculation of $h$ and $X$ for the one-dimensional cubic oscillator (3) yields $[15,16]$

$$
\begin{aligned}
h= & \frac{p^{2}}{2 m}+\frac{1}{2} \mu^{2} x^{2}+\frac{3}{2 \mu^{4}}\left(\frac{1}{m}\left\{x^{2}, p^{2}\right\}+\mu^{2} x^{4}+\frac{2 \hbar^{2}}{3 m}\right) \epsilon^{2} \\
+ & \mathcal{O}\left(\epsilon^{4}\right), \\
X= & x+\frac{2 i}{m \mu^{4}}\left(p^{2}+\frac{1}{2} m \mu^{2} x^{2}\right) \epsilon+\frac{1}{m \mu^{6}}\left(\left\{x, p^{2}\right\}\right. \\
& \left.-m \mu^{2} x^{3}\right) \epsilon^{2}+\mathcal{O}\left(\epsilon^{3}\right),
\end{aligned}
$$

where $\{\cdot, \cdot\}$ stands for the anticommutator. We can attempt to calculate the generating functional for this system using its Hermitian representation. This amounts to using $h-J x$ in place of $H-J X$ in (16). As shown in [14] this is already a very difficult task even in the absence of the source term [17]. If we try to use (16) to compute $Z[J]$ directly, we need to insert

$$
\begin{aligned}
H-J X= & \frac{p^{2}}{2 m}+\frac{\mu^{2} x^{2}}{2}-J x \\
& +\left[i x^{3}-\frac{2 i J}{m \mu^{4}}\left(p^{2}+\frac{1}{2} m \mu^{2} x^{2}\right)\right] \epsilon \\
& +\left[-\frac{J}{m \mu^{6}}\left(\left\{x, p^{2}\right\}-m \mu^{2} x^{3}\right)\right] \epsilon^{2}+\mathcal{O}\left(\epsilon^{3}\right)
\end{aligned}
$$

in (16) and try to obtain a Hamiltonian path-integral formula for $Z[J]$. Because up to terms of order $\epsilon^{2}, H-J X$ is quadratic in $p, Z[J]$ admits a Lagrangian path-integral formula that reads

$$
Z[J]=\int \mathcal{D}(x) e^{i / \hbar \int_{t_{1}}^{t_{2}} d t L(x, \dot{x} ; J)},
$$

where

$$
\begin{gathered}
\int \mathcal{D}(x):=\lim _{N \rightarrow \infty} \int_{-\infty}^{\infty} \cdots \int_{-\infty}^{\infty} \prod_{n=1}^{N} \mathfrak{m}\left(x_{n-1}\right) d x_{n}, \\
\mathfrak{m}(x):=\left[2 \pi i \hbar\left(t_{2}-t_{1}\right) N^{-1} g(x, J)\right]^{-(1 / 2)},
\end{gathered}
$$

$$
\begin{gathered}
L(x, \dot{x} ; J):=\frac{[\dot{x}-a(J)]^{2}}{2 g(x, J)}-v(x, J)+\mathcal{O}\left(\epsilon^{3}\right), \\
g(x, J):=m^{-1}\left[1-4 i \mu^{-4} J \epsilon-4 \mu^{-6} \epsilon^{2} x\right], \\
a(J):=-2 i \hbar m^{-1} \mu^{-6} J \epsilon^{2},
\end{gathered}
$$

$v(x, J):=\frac{1}{2} \mu^{2} x^{2}-J x+\left(i x^{3}-i \mu^{-2} J x^{2}\right) \epsilon-\mu^{-4} J x^{3} \epsilon^{2}$.

The appearance of $g(x, J)$ in expression (21) for the measure of the path-integral and the kinetic part of the Lagrangian (22) is reminiscent of the perturbative equivalence of this model and one described by a Hermitian effective position-dependent-mass Hamiltonian [15,18]. It is also worth mentioning that for $J \neq 0$ the computation of the path integral (19) is as difficult as the one obtained in the Hermitian description of the model that is based on (11).

Finally, we consider the non-Hermitian Hamiltonians of the form

$$
H_{\alpha}=\frac{p^{2}}{2 m}+V(x+i \alpha),
$$

where $V: \mathbb{R} \rightarrow \mathbb{R}$ is a real analytic function and $\alpha \in \mathbb{R}$. Clearly (26) is obtained through an imaginary shift of the coordinate $x$ in the Hermitian Hamiltonian $H_{0}$ [19]. If $V$ is taken to be an even function, (26) gives rise to a large class of $\mathcal{P} \mathcal{T}$-symmetric Hamiltonians:

$$
\begin{aligned}
H & =\frac{p^{2}}{2 m}+\sum_{n=0}^{\infty} c_{n}(x+i \alpha)^{2 n} \\
& =\frac{p^{2}}{2 m}+\sum_{n=0}^{\infty}(-1)^{n} c_{n}(i x-\alpha)^{2 n},
\end{aligned}
$$

where $c_{n}$ are real coefficients. A well-known example is [3]

$$
\begin{aligned}
H & =\frac{p^{2}}{2 m}+\frac{\mu^{2} x^{2}}{2}+i \epsilon x \\
& =\frac{p^{2}}{2 m}+\frac{\mu^{2}}{2}\left(x+i \mu^{-2} \epsilon\right)^{2}+\frac{\epsilon^{2}}{2 \mu^{2}} .
\end{aligned}
$$

Another example is the following three-parameter family of $\mathcal{P} \mathcal{T}$-symmetric Hamiltonians:

$$
H=\frac{p^{2}}{2 m}+a x^{4}+i b x^{3}+c x^{2}+\left[\frac{b\left(8 a c-b^{2}\right)}{16 a^{2}}\right](i x),
$$

with $a, b, c \in \mathbb{R}$ and $a \neq 0$, that is obtained from (27) by setting $\alpha=\frac{b}{4 \alpha}, c_{2}=a, c_{1}=c+\frac{3 b^{2}}{8 a}, c_{0}=\frac{b^{2}\left(16 a c+5 b^{2}\right)}{256 a^{3}}$, and $c_{n}=0$ for $n \geq 3$.

For the case that $V$ is not an even function the Hamiltonians (26) are not $\mathcal{P} \mathcal{T}$-symmetric. Nevertheless they satisfy (for general $V$ ) the pseudo-Hermiticity condi- 
tion [2]

$$
H_{\alpha}^{\dagger}=\eta_{\alpha} H_{\alpha} \eta_{\alpha}^{-1}
$$

for

$$
\eta_{\alpha}:=e^{2 \alpha p / \hbar}
$$

Because $\eta_{\alpha}$ is positive, $H_{\alpha}$ is equivalent to the Hermitian Hamiltonian [11]

$$
\begin{aligned}
h & =\eta_{\alpha}^{1 / 2} H_{\alpha} \eta_{\alpha}^{-(1 / 2)}=e^{\alpha p / \hbar} H_{\alpha} e^{-(\alpha p / \hbar)} \\
& =\frac{p^{2}}{2 m}+V\left(e^{\alpha p / \hbar} x e^{-(\alpha p / \hbar)}+i \alpha\right)=\frac{p^{2}}{2 m}+V(x)=H_{0} .
\end{aligned}
$$

In particular, the Hamiltonians (28) and (29) are, respectively, unitary equivalent to the harmonic oscillator Hamiltonian $h=\frac{p^{2}}{2 m}+\frac{\mu^{2} x^{2}}{2}$ and the quartic anharmonic oscillator Hamiltonian $h=\frac{p^{2}}{2 m}+a x^{4}+c x^{2}$.

The unitary equivalence of $H_{\alpha}$ and $H_{0}$ shows that the generating functional $Z[J]$ for $H_{\alpha}$ does not depend on $\alpha$, i.e.,

$$
\begin{aligned}
Z[J] & =\int_{-\infty}^{\infty} d x\left\langle x\left|e^{-\left(i\left(t_{2}-t_{1}\right)\left(H_{\alpha}-J X\right) / \hbar\right)}\right| x\right\rangle \\
& =\int_{-\infty}^{\infty} d x\left\langle x\left|e^{-\left(i\left(t_{2}-t_{1}\right)\left(H_{0}-J x\right) / \hbar\right)}\right| x\right\rangle .
\end{aligned}
$$

Because of the simple form of the metric operator, we can easily compute

$$
X=\eta_{\alpha}^{-(1 / 2)} x \eta_{\alpha}^{1 / 2}=x+i \alpha .
$$

Therefore, according to the first equation in (33),

$$
Z[J]=e^{i\left(t_{2}-t_{1}\right) \alpha J / \hbar}\left[\int_{-\infty}^{\infty} d x\left\langle x\left|e^{-\left(i\left(t_{2}-t_{1}\right)\left(H_{\alpha}-J x\right) / \hbar\right)}\right| x\right\rangle\right] .
$$

This shows that if one uses the incorrect expression (4) for $Z[J]$ that identifies the source term with $J x$ rather than $J X$, one misses the factor $e^{i\left(t_{2}-t_{1}\right) \alpha J / \hbar}$ on the right-hand side of (35). The resulting formula for $Z[J]$ will then depend on $\alpha$ and consequently contradict (33).

In summary, we showed that one can use the standard expression for the generating functional $Z[\vec{J}]$ in pseudoHermitian and in particular $\mathcal{P} \mathcal{T}$-symmetric quantum mechanics provided that one modifies the source term $\vec{J} \cdot \vec{x}$ to $\vec{J} \cdot \vec{X}$, where $\vec{X}$ is the pseudo-Hermitian position operator. The metric operator $\eta_{+}$that plays a central role in the operator formulation of the theory enters in the pathintegral expression for $Z[\vec{J}]$ through the source term, because $\vec{X}$ depends on $\eta_{+}$. The metric dependence of $\vec{X}$ is at the root of the difficulty in performing the calculations of $Z[\vec{J}]$ and the $n$-point functions of the theory.
[1] A. Mostafazadeh and A. Batal, J. Phys. A 37, 11645 (2004); A. Mostafazadeh, J. Phys. A 38, 3213 (2005).

[2] A. Mostafazadeh, J. Math. Phys. (N.Y.) 43, 205 (2002).

[3] C. M. Bender and S. Boettcher, Phys. Rev. Lett. 80, 5243 (1998).

[4] C. M. Bender, D. C. Brody, and H. F. Jones, Am. J. Phys. 71, 1095 (2003).

[5] C. M. Bender, I. Cavero-Pelaez, K. A. Milton, and K. V. Shajesh, Phys. Lett. B 613, 97 (2005); C. M. Bender, H. F. Jones, and R. J. Rivers, Phys. Lett. B 625, 333 (2005).

[6] H.F. Jones and R. J. Rivers, Phys. Rev. D 75, 025023 (2007).

[7] V. Jakubský, Mod. Phys. Lett. A 22, 1075 (2007).

[8] S. Weinberg, The Quantum Theory of Fields (Cambridge University Press, Cambridge, 1995), Vol. I.

[9] B. DeWitt, The Global Approach to Quantum Field Theory (Oxford University Press, Oxford, 2003).

[10] M. Reed and B. Simon, Functional Analysis (Academic Press, San Diego, 1980), Vol. I.
[11] A. Mostafazadeh, J. Phys. A 36, 7081 (2003).

[12] A. Mostafazadeh, "Time-Dependent Pseudo-Hermitian Hamiltonians Defining a Unitary Quantum System and Uniqueness of the Metric Operator" (unpublished).

[13] A. Mostafazadeh, Nucl. Phys. B640, 419 (2002).

[14] C. M. Bender, H.-H. Chen, and K. A. Milton, J. Phys. A 39, 1657 (2006); See also C. M. Bender, S. Boettcher, P. N. Meisinger, and Q. Wang, Phys. Lett. A 302, 286 (2002).

[15] A. Mostafazadeh, J. Phys. A 38, 6557 (2005).

[16] H. F. Jones, J. Phys. A 38, 1741 (2005).

[17] But it makes it easy to see that the leading order term in $Z[J]$ must be of order $\epsilon^{2}$.

[18] B. Bagchi, C. Quesne, and R. Roychoudhury, J. Phys. A 39, L127 (2006).

[19] M. Znojil, F. Cannata, B. Bagchi, and R. Roychoudhury, Phys. Lett. B 483, 284 (2000); Z. Ahmed, Phys. Lett. A 290, 19 (2001). 